\title{
GAIA Level 2a Small for Gestational Age
}

National Cancer Institute

\section{Source}

National Cancer Institute. GAlA Level 2a Small for Gestational Age. NCI Thesaurus. Code C128734.

GAIA Level 2a Small for Gestational Age is defined by three criteria: first, weight below the 10th percentile for gestational age; second, newborn weighed within 24 hours of birth on any scale with a less than or equal to $50 \mathrm{~g}$ resolution, calibrated and tared to zero; third, the gestational age is assessed by either a) Certain LMP with first or second trimester ultrasound; or b) Certain LMP with first trimester physical exam. 